\title{
Creation of a Claudin-2 Binder and Its Tight Junction-Modulating Activity in a Human Intestinal Mode|s
}

\author{
Mutsumi Takigawa, ${ }^{1}$ Manami lida, ${ }^{1}$ Shotaro Nagase, Hidehiko Suzuki, Akihiro Watari, \\ Minoru Tada, Yoshiaki Okada, Takefumi Doi, Masayoshi Fukasawa, Kiyohito Yagi, \\ Jun Kunisawa, and Masuo Kondoh
}

Graduate School of Pharmaceutical Sciences, Osaka University, Osaka, Japan (Mu.T., M.I., S.N., A.W., Y.O., T.D., K.Y., J.K., M.K.); Laboratory of Vaccine Materials and Laboratory of Gut Environmental System, National Institute of Biomedical Innovation, Osaka, Japan (Mu.T., H.S., J.K.); Division of Biological Chemistry and Biologicals, National Institutes of Health Sciences, Tokyo, Japan (Mi.T.); Department of Biochemistry and Cell Biology, National Institute of Infectious Diseases, Tokyo, Japan (M.F.); Graduate School of Medicine and Graduate School of Dentistry, Osaka University, Osaka, Japan (J.K.); Division of Mucosal Immunology, Department of Microbiology and Immunology and International Research and Development Center for Mucosal Vaccines, The Institute of Medical Science, The University of Tokyo, Tokyo, Japan (J.K.); and Department of Microbiology and Immunology, Kobe University Graduate School of Medicine, Hyogo, Japan (J.K.)

Received April 15, 2017; accepted August 31, 2017

\section{ABSTRACT}

Disruption of the gastrointestinal epithelial barrier is a hallmark of chronic inflammatory bowel diseases (IBDs). The transmembrane protein claudin 2 (CLDN2) is a component of epithelial tight junctions (TJs). In the intestines of patients with IBDs, the expression of the pore-forming TJ protein CLDN2 is upregulated. Although CLDN2 is involved in these leaky barriers, whether it can be a target to enhance TJ integrity is unknown because a CLDN2-specific inhibitor has not been developed. Here, we used DNA immunization to generate a monoclonal antibody $(m A b)$ that recognized an extracellular loop of CLDN2. Treatment of epithelial cell monolayers with the $\mathrm{mAb}$ increased barrier integrity. In addition, the anti-CLDN2 mAb attenuated the decrease in TJ integrity induced by the proinflammatory cytokine tumor necrosis factor- $\alpha$ (TNF- $\alpha$ ), and cotreatment of cells with anti-TNF- $\alpha$ mAb and anti-CLDN2 mAb showed additive attenuating effects. These findings indicate that CLDN2 may be a target for enhancing TJ integrity, and CLDN2 binder may be an enhancer of mucosal barrier integrity and a potential therapeutic option for IBDs.

\section{Introduction}

Inflammatory bowel diseases (IBDs), which include Crohn's disease and ulcerative colitis, are characterized by chronic relapsing idiopathic inflammation of the gastrointestinal tract that is associated with long-term and sometimes irreversible impairment of gastrointestinal structure and function (Podolsky, 2002). Patients with IBDs suffer from

This work was supported by a Health and Labour Sciences Research Grant from the Ministry of Health, Labour, and Welfare of Japan; a grant from the Japan Agency for Medical Research and Development; the Ministry of Education, Culture, Sports, Science, and Technology of (MEXT) of Japan/Japan Society for the Promotion of Science KAKENHI grant number 16H01373; funds from the Adaptable and Seamless Technology Transfer Program through Target-driven R\&D, Japan Science and Technology Agency; the Platform for Drug Discovery, Informatics, and Structural Life Science from MEXT of Japan; the Takeda Science Foundation; the Advanced Research for Medical Products Mining Program of the National Institute of Biomedical Innovation; the Science and Technology Research Promotion Program for Agriculture, Forestry, Fisheries, and Food Industry; and the Nipponham Foundation for the Future of Food.

${ }^{1} \mathrm{Mu} . \mathrm{T}$. and M.I. equally contributed to this study.

https://doi.org/10.1124/jpet.117.242214.

S This article has supplemental material available at jpet.aspetjournals.org. inflammation-induced "leak flux" diarrhea caused by a passive loss of ions and water from the circulation into the intestinal lumen as a result of an impaired intestinal barrier (Hering et al., 2012). The chronic intestinal inflammation is induced by multiple exogenous and endogenous signals and mediated by various immune cells (de Souza and Fiocchi, 2016). The exogenous substances include dietary antigens, gut microbiotaderived microbe-associated molecular patterns, pathogens, and xenobiotics, all of which undergo epithelial translocation and thus activate the intestinal immune responses in patients with IBDs (de Souza and Fiocchi, 2016). Therefore, one therapeutic strategy for IBDs is to suppress inflammation, and anti-inflammatory agents including steroids, thiopurines, methotrexate, and monoclonal antibodies to tumor necrosis factor $\alpha$ (TNF- $\alpha$ ) have been incorporated into treatment regimens (de Souza and Fiocchi, 2016). However, many patients have to stop these therapies because of adverse drug effects or lack of therapeutic responses (Bravatà et al., 2015).

Despite the role of epithelial translocation of exogenous substances in the activation of intestinal immune responses in IBDs (de Souza and Fiocchi, 2016), therapeutic interventions

ABBREVIATIONS: CLDN, claudin; FITC, fluorescein isothiocyanate; hCLDN, human claudin; IBD, inflammatory bowel disease; mAb, monoclonal antibody; mCLDN, mouse claudin; MDCKI, Madin-Darby canine kidney I; PBS, phosphate-buffered saline; PI3K, phosphatidylinositol-3-kinase; TEER, transepithelial electrical resistance; TJ, tight junction; TNF- $\alpha$, tumor necrosis factor $\alpha$. 
targeted at the epithelial translocation have never been fully developed.

Chronic inflammation in IBDs compromises the intestinal epithelial monolayer. Concentrations of the proinflammatory cytokine TNF- $\alpha$ are increased in Crohn's disease and ulcerative colitis (Podolsky, 2002). To maintain the barrier function of the intestinal epithelium, its intercellular spaces are sealed by tight junctions (TJs), which prevent the free movement of solutes (ions, water, and noxious antigens derived from foods or microorganisms) across epithelial sheets (Powell, 1981). The intercellular TJ seal is impaired in colonic samples from IBD patients (Zeissig et al., 2007), and TNF- $\alpha$ plays a pivotal role in the disruption of TJ seals, perhaps through altered regulation of TJ components (Schmitz et al., 1999; Bürgel et al., 2002; Amasheh et al., 2009; Mankertz et al., 2009).

Freeze-fracture analysis showed that TJ seals consist of a set of continuous, anastomosing intramembranous strands (Staehelin, 1974). TJs comprise transmembrane proteins [junctional adhesion molecules, occludin, claudins (CLDNs), and tricellulin] and intracellular scaffolding proteins (zonula occludens) (Van Itallie and Anderson, 2014). CLDNs are key components of the TJ seal. CLDNs are a family containing 27 members that show tissue-specific expression profiles. CLDN1, 3, 4, 5, 7, and 8 are frequently observed in tight epithelia, whereas CLDN2 is primarily expressed in leaky epithelia (Luettig et al., 2015). Colon biopsy samples from patients with IBDs showed decreased expression of CLDN3, 5, and 8 and occludin and increased expression of CLDN2 (Zeissig et al., 2007).

CLDN2 is a $24.5-\mathrm{kDa}$ tetra-transmembrane protein with two extracellular loop domains (Furuse et al., 1998b). Transfection with a plasmid encoding CLDN2 decreases the integrity of TJs, due to the formation of discontinuous TJ strands (Furuse et al., 2001). Treatment with TNF- $\alpha$ upregulates the expression of CLDN2 in part via phosphatidylinositol3-kinase (PI3K) signaling (Prasad et al., 2005; Heller et al., 2008; Mankertz et al., 2009; Amasheh et al., 2010; Suzuki et al., 2011), whereas anti-TNF- $\alpha$ monoclonal antibody (mAb) prevents the TNF- $\alpha$-induced loss of TJ integrity and the associated downstream effects on PI3K and CLDN2 (Fischer et al., 2013). Furthermore, the administration of an anti-TNF- $\alpha$ antibody restores intestinal barrier function in patients with Crohn's disease (Suenaert et al., 2002; Noth et al., 2012). These findings indicate that the inhibition of CLDN2 may restore the diminished TJ integrity in IBDs. However, whether CLDN2 is a feasible target for IBD therapy has been unknown because a CLDN2-specific inhibitor had not been developed.

In this study, we used a DNA immunization method to generate a mAb that specifically recognizes the first extracellular loop of CLDN2, and we investigated the effects of this $\mathrm{mAb}$ on TNF- $\alpha$-induced loss of TJ integrity in cell culture models.

\section{Materials and Methods}

Reagents. Anti-histidine tag $\mathrm{mAb}$, fluorescein isothiocyanate (FITC)-conjugated antibody, rat IgG, anti- $\beta$-actin antibody, and TNF- $\alpha$ were obtained from Thermo Fisher Scientific (Waltham, MA), Jackson ImmunoResearch (West Grove, PA), BD Biosciences (Franklin Lakes, NJ), Sigma-Aldrich (St. Louis, MO), and R\&D Systems (Minneapolis, MN), respectively. Anti-CLDN1 mAb (7A5),
anti-CLDN4 mAb (5D12), and anti-CLDN3 and -4 mAb (5A5) were prepared as described previously (Fukasawa et al., 2015; Kuwada et al., 2015). The anti-TNF- $\alpha$ monoclonal antibodies infliximab (Remicade; Mitsubishi Tanabe Pharma Corporation, Osaka, Japan) and adalimumab (Humira; Eisai, Tokyo, Japan) were purchased from reagent distributors.

Cell Cultures. Because they are CLDN negative, HT1080 cells (a human fibrosarcoma cell line) are used to characterize CLDN binders (Neesse et al., 2013; Fukasawa et al., 2015; Mosley et al., 2015; Nakajima et al., 2015). HT1080 cells mock transfected or stably transfected with human CLDN1-CLDN7 or CLDN9 (mock/HT1080, hCLDN1/HT1080 to hCLDN7/HT1080, and hCLDN9/HT1080 cells, respectively) were previously established ( $\mathrm{Li}$ et al., 2014). hCLDN/HT1080 cells were grown in Dulbecco's modified Eagle's medium containing $10 \%$ fetal bovine serum at $37^{\circ} \mathrm{C}$ under $5 \% \mathrm{CO}_{2}$.

L cells that stably expressed mouse CLDN1 or CLDN2 (mCLDN1/L and $\mathrm{mCLDN} 2 / \mathrm{L}$ cells, respectively) were provided by Dr. S. Tsukita (Kyoto University, Kyoto, Japan). mCLDN/L cells were cultured in modified Eagle's medium containing $10 \%$ fetal bovine serum at $37^{\circ} \mathrm{C}$ under $5 \% \mathrm{CO}_{2}$.

Human intestinal epithelial cells (Caco-2; American Type Culture Collection, Manassas, VA) were grown in modified Eagle's medium containing $10 \%$ fetal bovine serum at $37^{\circ} \mathrm{C}$ under $5 \% \mathrm{CO}_{2}$.

Madin-Darby canine kidney I (MDCKI) cells (European Collection of Animal Cell Cultures, Porton Down, UK) were grown in Dulbecco's modified Eagle's medium containing $10 \%$ fetal bovine serum at $37^{\circ} \mathrm{C}$ under $5 \% \mathrm{CO}_{2}$.

Animals. Female Wistar rats (6 weeks of age) and female BALB/c Slc-nu/nu mice (8 weeks of age) were purchased from Shimizu Laboratory Supplies (Kyoto, Japan) and SLC, Inc. (Shizuoka, Japan), respectively. All animals were maintained under controlled conditions of a 12-hour light/dark cycle at $23 \pm 1.5^{\circ} \mathrm{C}$. The rodents had unrestricted access to food and water. All of the experimental protocols conformed to the ethics guidelines of the Graduate School of Pharmaceutical Sciences, Osaka University.

Preparation of a CLDN-Binding Peptide. We previously created $\mathrm{m} 19$, a peptide that binds to CLDNs $1,2,4$, and 5 , by using the CLDN3/4-binding polypeptide C-terminal fragment of Clostridium perfringens enterotoxin as a template (Takahashi et al., 2012). We then prepared m19 tagged with a histidine tag at the $\mathrm{N}$ terminus. Briefly, pET16b plasmid encoding m19 was transformed into Escherichia coli strain BL21 (DE3), and the production of the recombinant protein was induced by adding isopropyl-D-thiogalactopyranoside. Harvested cells were lysed in buffer A $[10 \mathrm{mM}$ Tris-HCl $(\mathrm{pH} 8.0)$, $400 \mathrm{mM} \mathrm{NaCl}, 5 \mathrm{mM} \mathrm{MgCl} 2,0.1 \mathrm{mM}$ phenylmethanesulfonyl fluoride, $1 \mathrm{mM} 2$-mercaptoethanol, and $10 \%$ glycerol]. Supernatants were applied to a HiTrap Chelating HP column (GE Healthcare, Little Chalfont, UK), and the recombinant protein was eluted with buffer A containing imidazole. The solvent was exchanged with phosphatebuffered saline (PBS) by using a PD-10 column (GE Healthcare), and the purified protein was stored at $-80^{\circ} \mathrm{C}$ until use. Purification of the recombinant protein was confirmed by SDS-PAGE followed by staining with Coomassie Brilliant Blue. Protein was quantified by using a BCA Protein Assay Kit (Pierce Biotechnology, Waltham, MA) and bovine serum albumin as a standard.

Generation of Anti-CLDN2 mAb. Female Wistar rats (6 weeks of age) were immunized with a eukaryotic expression vector encoding human CLDN2 every 2 weeks for 2 months according to proprietary technology (Genovac, Freiburg, Germany). Lymphocytes were removed 7 days after the last immunization and fused with P3UI cells in the presence of polyethylene glycol 1000, thus generating hybridoma cells. Hybridoma cells producing anti-CLDN2 mAbs were initially screened for the ability of their conditioned medium to bind to hCLDN2/HT1080 cells but not to mock/HT1080 cells, resulting in the isolation of a hybridoma that produced mAb against hCLDN2 (clone 1A2). The Ig class and subclass of anti-CLDN2 1A2 were determined by using a rat Ig-isotyping enzyme-linked immunosorbent assay kit (BD Biosciences). 
Purification of mAb. According to standard procedures, female BALB/c Slc- $n u / n u$ mice (8 weeks of age; Japan SLC, Inc., Hamamatsu, Japan) were each intraperitoneally injected with the adjuvant pristane (Sigma-Aldrich) and $1 \times 10^{7} \mathrm{mAb}$-producing hybridoma cells. Ascites was collected, and the $\mathrm{mAb}$ was purified by using a protein $\mathrm{G}$ column (GE Healthcare). Eluted antibodies were dialyzed in PBS. The concentration of the purified antibody was determined by measuring the absorbance at $280 \mathrm{~nm}$.

Flow Cytometry. hCLDN/HT1080 or mCLDN/L cells $\left(5 \times 10^{5}\right.$ cells) were incubated with PBS containing $1 \%$ bovine serum, m19 $(10 \mu \mathrm{g} / \mathrm{ml})$, or mAbs $(5 \mu \mathrm{g} / \mathrm{ml})$ for 1 hour at $4^{\circ} \mathrm{C}$. Cells treated with $\mathrm{m} 19$ were then treated with an anti-histidine tag $\mathrm{mAb}(5 \mu \mathrm{g} / \mathrm{ml}$; Thermo Fisher Scientific). The cells were then incubated with FITCconjugated secondary antibody ( $10 \mu \mathrm{g} / \mathrm{ml}$; Jackson ImmunoResearch) and stained with propidium iodide solution ( $1 \mu \mathrm{g} / \mathrm{ml}$; Miltenyi Biotec, Bergisch Gladbach, Germany) to exclude dead cells from flow cytometric analysis. The mAb-bound cells were detected with a FACSCalibur Flow Cytometer (BD Biosciences), and data were analyzed by using CellQuest Software (BD Biosciences).

Chimeric CLDN2/HT1080 Cells. In CLDN2 cDNA, the sequences encoding the first extracellular loop domain (corresponding to amino acids 28 through 78 ) of hCLDN2, the second extracellular loop domain (corresponding to amino acids 144 through 162) of hCLDN2, or the first and second loop domains of hCLDN2 were genetically replaced by those of hCLDN4, resulting in hCLDN2/EL1-hCLDN4, hCLDN2/EL2-hCLDN4, or hCLDN2/EL1 and EL2-hCLDN4 cDNA. The resultant cDNAs were cloned into pcDNA3.1(-) (Invitrogen, Carlsbad, CA). The chimeric hCLDN2 expression vectors were then transfected into HT1080 cells by using $\mathrm{X}$-tremeGENE HP DNA transfection reagent (Roche Diagnostics, Basel, Switzerland), and G418-resistant clones (stable transfectants for each chimeric hCLDN2) were isolated. Chimeric hCLDN/HT1080 cells were maintained in Dulbecco's modified Eagle's medium containing $10 \%$ fetal bovine serum.

Assay of TJ Integrity. Caco-2 or MDCK I cells were recovered by gentle pipetting with trypsin until a single-cell suspension was obtained, and these cells were seeded into 24-well Transwell inserts (Corning Life Sciences, Tewksbury, MA) at a density of $6.0 \times 10^{4}$ or $5.0 \times 10^{4}$ cells per $0.33-\mathrm{cm}^{2}$ well, respectively. The monolayers were then cultured for 10-14 days before basolateral treatment with mAb (0.01-10 $\mu \mathrm{g} / \mathrm{ml}$ ). Transepithelial electrical resistance (TEER) during treatment was measured by using a Millicell-ERS Epithelial VoltOhm Meter (Millipore, Billerica, MA). The background TEER value of a well containing medium only was subtracted from the recorded TEER; these values were then normalized to the surface area of the well.

Assay of TJ Integrity in TNF- $\alpha$-Treated Caco-2 Monolayers. Caco-2 cells were seeded into 24-well Transwell inserts $\left(6.0 \times 10^{4}\right.$ cells $/ 0.33-\mathrm{cm}^{2}$ well; Corning Life Sciences). The Caco- 2 monolayers were then cultured for 10 days until the TEER value had reached a plateau, and the cells were used for various analyses.

To investigate the effects of anti-CLDN2 mAb on TNF- $\alpha$-induced reduction of TJ integrity, the cells were cotreated with TNF- $\alpha$ ( $10 \mathrm{ng} / \mathrm{ml}$; R\&D Systems) and either rat IgG (10 $\mu \mathrm{g} / \mathrm{ml}$; R\&D Systems) or anti-CLDN2 mAb $(10 \mu \mathrm{g} / \mathrm{ml})$. TEER values were measured 24 hours before and 24 hours after treatment. Alternatively, the Caco- 2 cells were pretreated with TNF- $\alpha(10 \mathrm{ng} / \mathrm{ml})$ for 24 hours, and then rat IgG $(10 \mu \mathrm{g} / \mathrm{ml})$ or anti-CLDN2 mAb 1A2 $(10 \mu \mathrm{g} / \mathrm{ml})$ was added; the cells were cultured, and TEER values were measured.

To investigate the effects of anti-TNF- $\alpha$ mAb and anti-CLDN2 mAb, the cells were pretreated with TNF- $\alpha(10 \mathrm{ng} / \mathrm{ml})$ for 24 hours, after which anti-TNF- $\alpha$ mAb (infliximab or adalimumab), anti-CLDN2 $\mathrm{mAb} 1 \mathrm{~A} 2$, or a mixture of anti-TNF- $\alpha \mathrm{mAb}$ and anti-CLDN2 mAb $1 \mathrm{~A} 2$ was added to the medium $(10 \mu \mathrm{g} / \mathrm{ml} /$ reagent). TEER values were measured after treatment.

Immunoblot Analysis. MDCK I or Caco-2 cells were collected by using a cell scraper and lysed in buffer $(50 \mathrm{mM}$ Tris-HCl, $150 \mathrm{mM}$ $\mathrm{NaCl}, 0.1 \%$ SDS, $1 \% \mathrm{NP} 40,0.5 \%$ sodium deoxycholate, and $1 \%$ protease inhibitor cocktail; Nacalai Tesque, Kyoto, Japan). The resultant supernatants were recovered by centrifugation. Lysates (10 $\mu \mathrm{g} / \mathrm{sample}$ ) underwent SDS-PAGE in a $15 \%$ polyacrylamide gel; the separated proteins were blotted onto a polyvinylidene difluoride membrane. Membranes were incubated with Tris-buffered saline containing $0.1 \%(\mathrm{v} / \mathrm{v})$ Tween-20 and $2 \%(\mathrm{v} / \mathrm{v})$ bovine serum albumin for 2 hours and then incubated with the primary antibody for 1 hour [anti-CLDN2 at $0.5 \mu \mathrm{g} / \mathrm{ml}$ (Invitrogen) or anti- $\beta$-actin at $0.4 \mu \mathrm{g} / \mathrm{ml}$ (Sigma-Aldrich)]. After several washes in Tris-buffered saline containing $0.1 \%$ Tween-20, the membrane was incubated with a horseradish peroxidase-conjugated secondary antibody (goat anti-mouse IgG at $0.5 \mu \mathrm{g} / \mathrm{ml}$; Millipore) for 1 hour. Immunoreactive bands were detected by using a chemiluminescence reagent (Nacalai Tesque) and an image analyzer (LAS 4010; GE Healthcare).

Statistical Analysis. Data were analyzed by using Tukey's test. The statistical significance for all comparisons was set at $P<0.05$.

\section{Results}

Generation of Anti-CLDN2 mAbs. CLDN2 forms homodimers with adjacent CLDN2 or heterodimers with CLDN3, but does not interact with CLDN1 (Furuse et al., 1999), and singlemolecule force spectroscopy revealed that these trans-interactions are mediated through the first extracellular loop domain of CLDN2 (Lim et al., 2008). We therefore hypothesized that a molecule that binds to an extracellular domain of CLDN2 would prevent the formation of leaky barriers caused by CLDN2, thus attenuating the CLDN2-mediated impairment of the $\mathrm{TJ}$ seal in IBDs. To address this hypothesis, we first tried to generate mAbs that recognized one or both of the extracellular loop domains of CLDN2. We previously generated anti-CLDN1 and anti-CLDN4 mAbs by immunizing mice and rats with plasmid DNA encoding hCLDN1 and hCLDN4 cDNA, respectively (Li et al., 2014; Fukasawa et al., 2015). Therefore, we immunized rats with plasmid DNA encoding hCLDN2 and selected hybridoma cells that produced anti-CLDN2 mAbs, which bound to hCLDN2/HT1080 cells but not to mock/HT1080 cells; these efforts resulted in the generation of a hybridoma clone that produced anti-CLDN2 mAb (1A2). The Ig subtype of $1 \mathrm{~A} 2$ is IgG2b. 1A2 bound to hCLDN2/HT1080 cells but not to HT1080 cells that stably expressed hCLDN1, 3, 4, 6, 7, or 9 (Fig. 1A); $1 \mathrm{~A} 2$ also bound to mCLDN2/L cells (Fig. 1A). These findings indicate that $\mathrm{mAb} 1 \mathrm{~A} 2$ is a specific binder of CLDN2.

To determine the extracellular domains to which 1A2 binds, we replaced either or both of the extracellular loop domains of CLDN2 with those of CLDN4. The broadly reactive CLDN binder $\mathrm{m} 19$, which binds to CLDNs $1-5$, bound to all of these chimeric CLDN2-expressing HT1080 cells (Fig. 1B). In contrast, mAb $1 \mathrm{~A} 2$ bound only to fractions containing the first extracellular domain of CLDN2 (Fig. 1B). Therefore, mAb 1A2 specifically recognizes the first extracellular loop domain of hCLDN2.

Effects of Anti-CLDN2 mAb on TJ Integrity. Monolayers of Caco-2 cells are widely used as an in vitro model of the human intestinal epithelial barrier (Meunier et al., 1995; Wang et al., 2005). Therefore, to assess whether a CLDN2 binder might attenuate the CLDN2-induced impairment of epithelial TJ integrity, we investigated the effects of antiCLDN2 mAb on TJ integrity in Caco-2 cell monolayers. mAb 1A2 increased TEER values to $133 \%$, and the removal of $1 \mathrm{~A} 2$ from the medium attenuated changes in TEER (Fig. 2A). Furthermore, 1A2 dose-dependently increased TEER (Fig. 2B). 


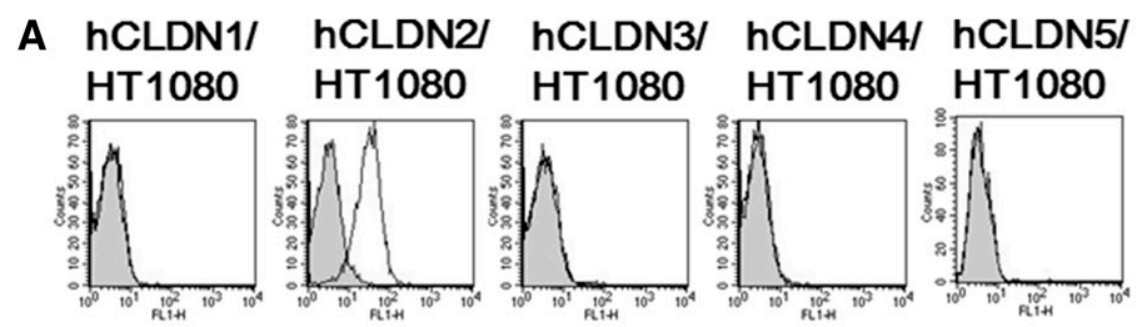

hCLDN6/ hCLDN7/ hCLDN9/ mCLDN1/ mCLDN2/ HT1080
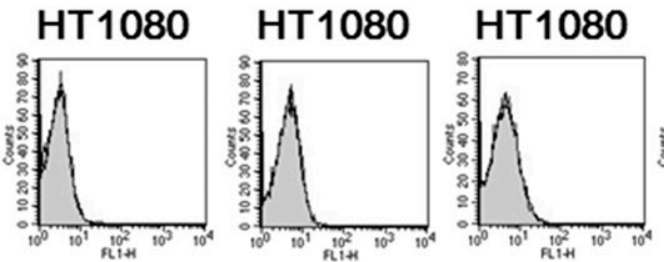

L cell

$\mathrm{L}$ cell

\section{Vehicle}

B

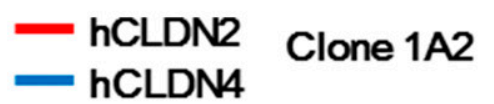

hCLDN2

EL1-

hCLDN4/

HT1080

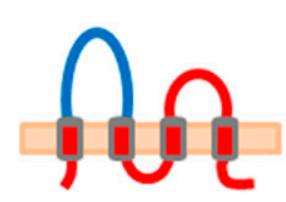

hCLDN2

EL2-

hCLDN4/

HT1080
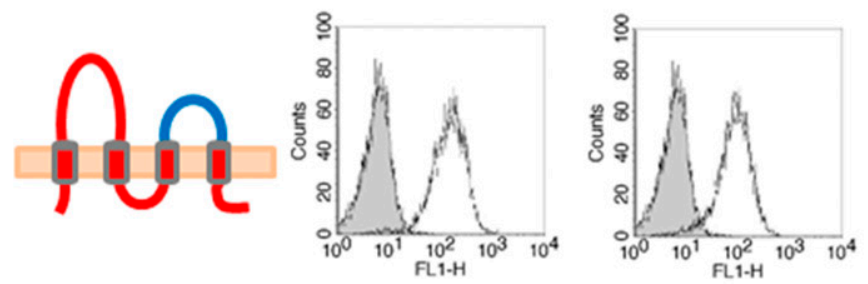

\section{hCLDN2}

EL1-EL2-

hCLDN4/

HT1080

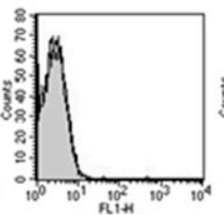

\section{Anti-CLDN2 mAb}
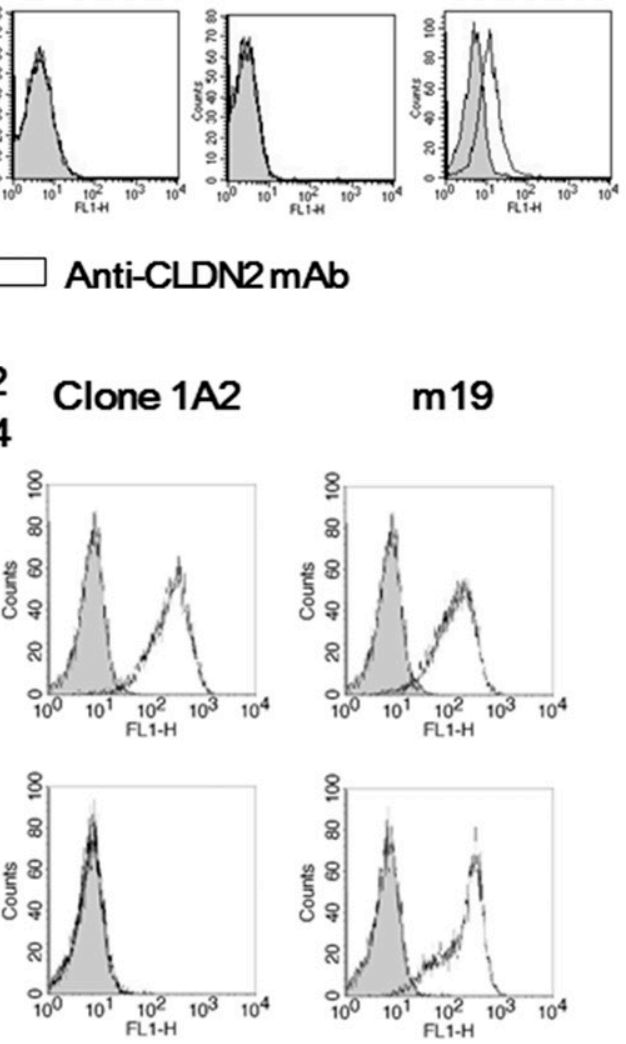

Fig. 1. Characterization of anti-CLDN2 mAb. (A) CLDN specificity of anti-CLDN2 mAb. hCLDN/HT1080 or mCLDN/L cells were incubated with rat anti-CLDN2 mAb (1A2) or PBS as a control, and then treated with goat anti-rat IgG $(\mathrm{H}+\mathrm{L})$ antibody conjugated with FITC. The antibodybound cells were detected by using a flow cytometer. Gray and white histograms represent vehicle-treated (with PBS) and antibody-treated cells, respectively. Data are representative of three independent experiments. (B) Epitope identification of anti-CLDN2 mAb. Either the first or second extracellular domain or both the first and second extracellular domains of hCLDN2 were genetically replaced by those of hCLDN4 to yield hCLDN2 EL1hCLDN4, hCLDN2 EL2-hCLDN4, or hCLDN2 EL1-EL2-hCLDN4, respectively. HT1080 cells transfected with hCLDN2, hCLDN4, or each chimeric CLDN2 were incubated with $5 \mu \mathrm{g} / \mathrm{ml}$ anti-CLDN2 mAb (white histogram), $10 \mu \mathrm{g} / \mathrm{ml}$ broad-specific CLDN binder ( $\mathrm{m} 19$; white histogram), or vehicle (PBS; gray histogram) followed by treatment of cells with FITC-conjugated goat anti-rat IgG $(\mathrm{H}+\mathrm{L})$ or anti- $6 \times$ His tag $\mathrm{mAb}$ and FITC-conjugated goat anti-mouse IgG. Cells that had bound anti-CLDN2 mAb or m19 were detected by using a flow cytometer. Data are representative of three independent experiments.
To confirm the CLDN2 specificity of the 1A2-induced increase in TJ integrity, we investigated the effects of mAbs specific for CLDN1 (7A5), CLDN4 (5D12), and CLDN3/4 (5A5) on TJ integrity (Li et al., 2014; Fukasawa et al., 2015).
Treatment of Caco-2 monolayers with mAb specific for CLDN1 (7A5) or CLDN4 (5D12) (10 $\mu \mathrm{g} / \mathrm{ml})$ did not significantly change TEER from that of the vehicle-only control, and treatment for 24 hours with the CLDN3/4 dual-specific mAb (5A5) decreased 

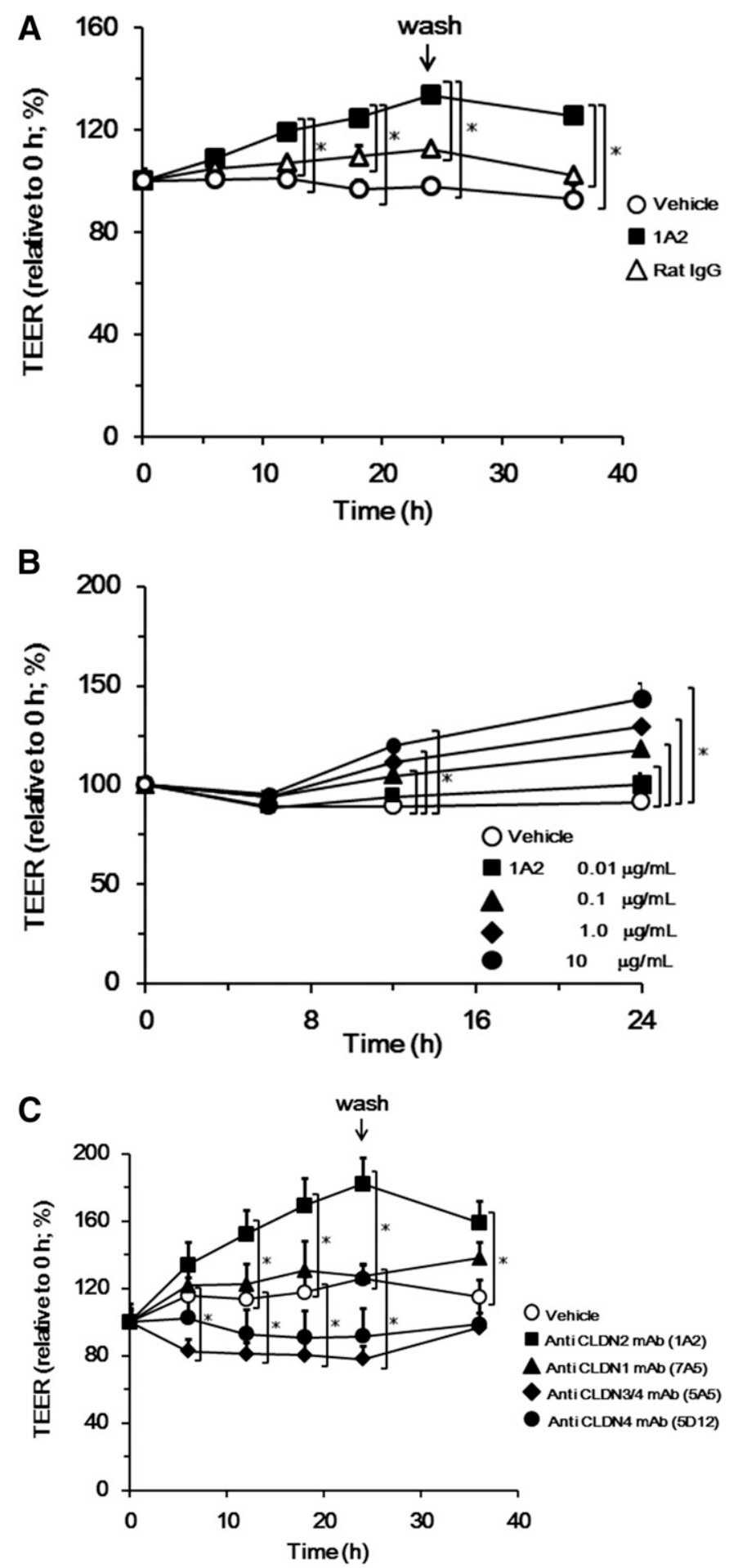

Fig. 2. Effects of anti-CLDN2 mAb on TJ seals in Caco-2 monolayers. (A) Effects of anti-CLDN2 mAb on TJ integrity. Caco-2 monolayers were cultured for 10 days and then treated with vehicle (PBS), rat IgG $(10 \mu \mathrm{g} / \mathrm{ml}$; as a negative control), or anti-CLDN2 $\mathrm{mAb}(1 \mathrm{~A} 2 ; 10 \mu \mathrm{g} / \mathrm{ml})$ on the basal side of the insert. The cells were exposed to the antibodies for 24 hours, washed with medium to remove antibodies, and cultured for an additional 12 hours. Changes in TEER values were monitored throughout treatment. Data are presented as the mean \pm S.D. $(n=3) . * P<0.05$ (Tukey's test). (B) Dose dependency of barrier-modulating activity of antiCLDN2 mAb in TEER. Caco-2 monolayers were cultured for 10 days and then treated with vehicle (PBS) or anti-CLDN2 mAb (1A2; 0.01, 0.1, 1 , or $10 \mu \mathrm{g} / \mathrm{ml}$ ) on the basal side of the insert. The cells were exposed to the antibodies for 24 hours. Changes in TEER values were monitored throughout treatment. Data are presented as the mean \pm S.D. $(n=3)$.
TEER to $78 \%$ of that of the vehicle control (Fig. 2C). In addition, we transfected MDCKI cells, which do not express CLDN2, to stably express CLDN2 and examined the effect of $\mathrm{mAb} 1 \mathrm{~A} 2$ on TJ integrity. Treatment with mAb $1 \mathrm{~A} 2$ increased TEER in MDCKI/CLDN2 cells but not MDCKI/vector cells (Supplemental Fig. 1). Similarly, mAb 1A2 induced a greater increase in the TEER of Caco-2 cells exogenously expressing CLDN2 compared with Caco-2/vector cells (Supplemental Fig. 2). Therefore, the ability of $1 \mathrm{~A} 2$ to promote TJ integrity might be due to specific interaction with CLDN2.

Effects of Anti-CLDN2 mAb on TNF- $\alpha$-Induced TJ Dysfunction. To investigate the effects of the anti-CLDN2 $\mathrm{mAb} 1 \mathrm{~A} 2$ on the disruption of TJ integrity in the context of inflammation, we used a Caco- 2 model of TNF- $\alpha$-induced TJ dysfunction (Ma et al., 2005; Graham et al., 2006). Treatment of cells with TNF- $\alpha$ (10 ng/ml) for 24 hours decreased TEER to $70 \%$ of that at 0 hour, accompanied by an increase in cellular CLDN2 protein content (Fig. 3A; Supplemental Fig. 3). In contrast, cotreatment with TNF- $\alpha$ and anti-CLDN2 mAb (1A2) attenuated the reduction in TEER (Fig. 3A). To clarify the effects of anti-CLDN2 mAb in the TNF- $\alpha$-induced dysfunction model, Caco-2 cells were exposed to TNF- $\alpha$ for 24 hours and then treated with anti-CLDN2 mAb for 48 hours. In cells exposed to TNF- $\alpha$ for 24 hours followed by 48-hour treatment with vehicle only or rat IgG, TEER decreased to $37 \%$ or $39 \%$, respectively, of that before TNF- $\alpha$ treatment (Fig. 3B). In contrast, anti-CLDN2 mAb treatment attenuated the TNF- $\alpha$-induced reduction in TEER to approximately $64 \%$ of that before TNF- $\alpha$ treatment.

To investigate whether the effects of mAbs against CLDN2 and TNF- $\alpha$ might be additive, we pretreated Caco- 2 cells with TNF- $\alpha$ (10 ng/ml) for 24 hours and then exposed the cells for 48 hours to anti-CLDN2 mAb (1A2), anti-TNF- $\alpha$ mAb (infliximab or adalimumab), or both anti-CLDN2 and anti-TNF- $\alpha$ mAbs concurrently. Pretreatment with TNF- $\alpha$ reduced TEER to approximately $48 \%$ of that of untreated cells (Fig. 3C). However, exposure to anti-CLDN2 mAb, infliximab, or adalimumab (10 $\mu \mathrm{g} / \mathrm{ml}$ each) for 48 hours recovered TEER to $75 \%$, $60 \%$, or $62 \%$ of the levels of cells before TNF- $\alpha$ treatment, respectively, and cotreatment with anti-CLDN2 mAb and either infliximab or adalimumab increased TEER to $93 \%$ and $85 \%$, respectively, of that of cells before TNF- $\alpha$ treatment. These findings indicate that CLDN2-targeted therapeutics might represent a new option in the treatment of IBDs.

\section{Discussion}

The activation of mucosal immune responses and impairment of the intestinal barrier are key characteristics of IBDs (Podolsky, 2002). Various attenuators of these immune responses, including steroids, thiopurines, methotrexate, and anti-TNF- $\alpha$ mAbs, are used clinically to treat patients with

${ }^{*} P<0.05$ (Tukey's test). (C) Effects of anti-CLDN1, anti-CLDN4, and antiCLDN3/4 mAbs on TJ integrity. Caco-2 monolayers were cultured for 10 days and then treated with vehicle (PBS), anti-CLDN1 mAb (7A5), antiCLDN2 mAb (1A2), anti-CLDN4 mAb (5D12), or anti-CLDN3/4 mAb (5A5) $(10 \mu \mathrm{g} / \mathrm{ml})$ on the basal side of the insert. The cells were exposed to the antibodies for 24 hours, washed with medium to remove antibodies, and cultured for an additional 12 hours. Changes in TEER values were monitored during antibody treatment. Data are presented as the mean \pm S.D. $(n=3)$. $* P<0.05$ (Tukey's test). 

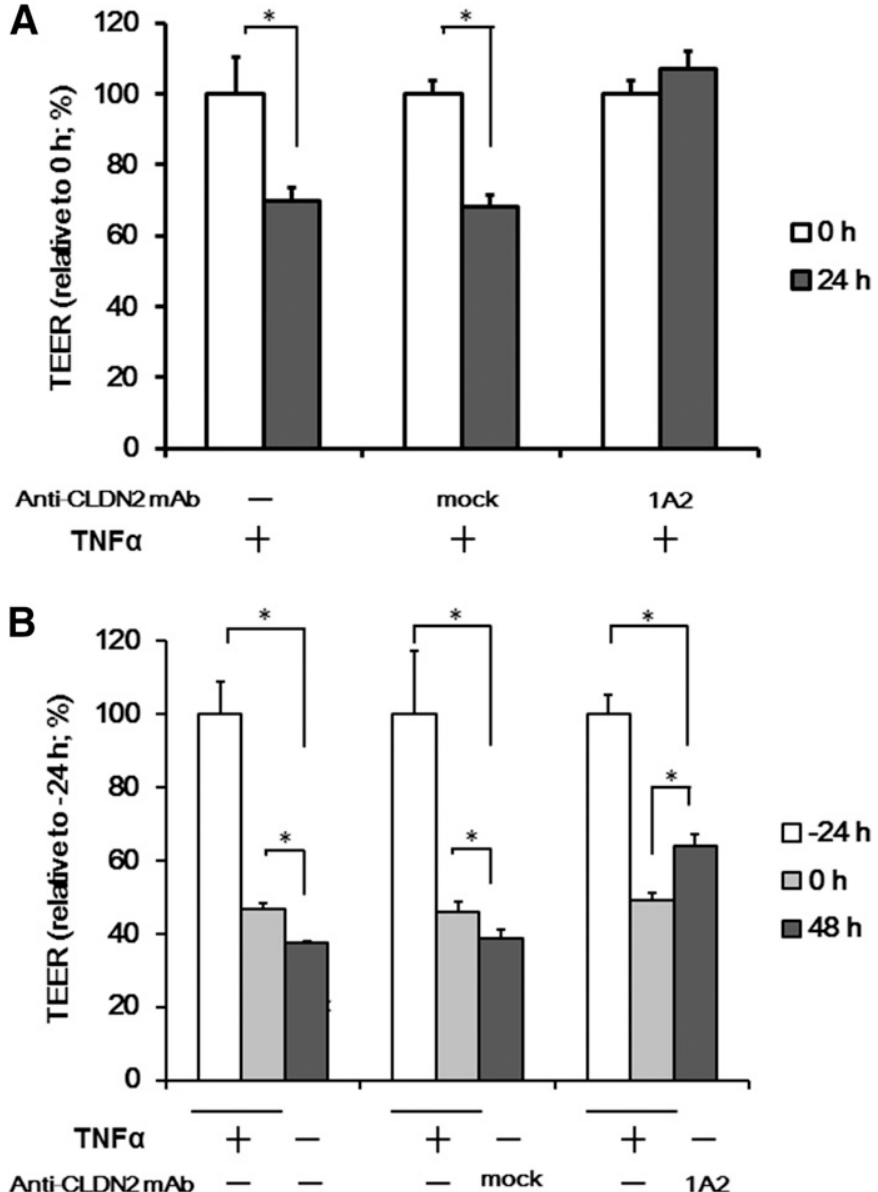

C

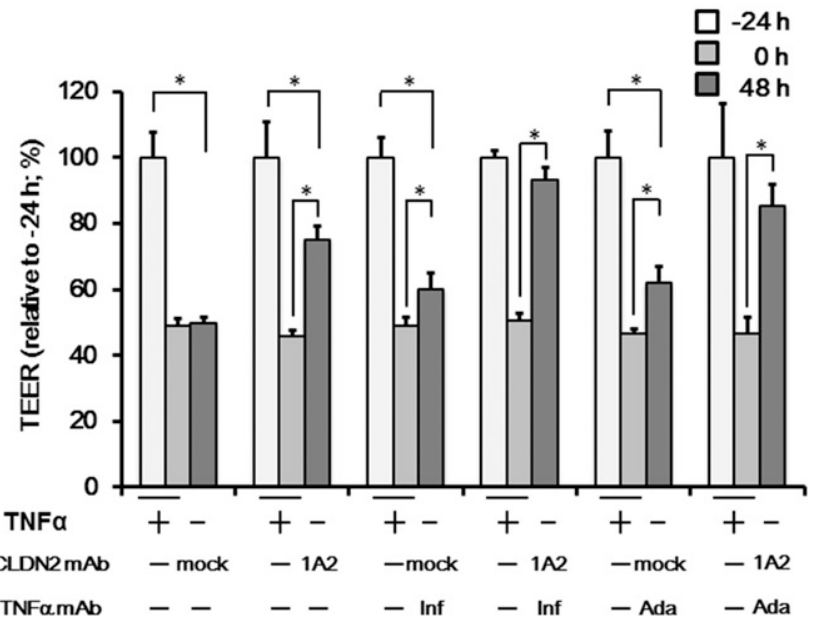

Fig. 3. Effects of anti-CLDN2 $\mathrm{mAb}$ on the disrupted TJ integrity in an in vitro model of IBDs. (A) Effects of anti-CLDN2 $\mathrm{mAb}$ on TJ dysfunction induced by TNF- $\alpha$ treatment. Once TEER values had reached a plateau (approximately 10 days after cells were seeded on the Transwell membrane), the cells were treated with vehicle (medium) or TNF- $\alpha$ $(10 \mathrm{ng} / \mathrm{ml})$ in the presence of vehicle (PBS), anti-CLDN2 mAb 1A2, or rat $\operatorname{IgG}(10 \mu \mathrm{g} / \mathrm{ml})$ for 24 hours. TEER values were measured at 0 hour (white bars) and 24 hours (gray bars) of treatment. Data are shown as the mean \pm S.D. $(n=3)$. $* P<0.05$ as determined by Tukey's test. (B) Effects of antiCLDN2 mAb on the TJ integrity disrupted by TNF- $\alpha$ treatment. When TEER values had reached a plateau (approximately 10 days after seeding on the Transwell membrane), the cells were treated with vehicle (medium) or TNF- $\alpha(10 \mathrm{ng} / \mathrm{ml})$ for 24 hours followed by treatment with vehicle (PBS), anti-CLDN2 $\mathrm{mAb} 1 \mathrm{~A} 2$, or rat IgG $(10 \mu \mathrm{g} / \mathrm{ml})$ for 48 hours. TEER values were measured at -24 hours (before treatment with TNF- $\alpha$, white bars), 0 hour (before treatment with anti-CLDN2 mAb, gray bars), and 48 hours
IBDs (de Souza and Fiocchi, 2016). However, therapeutic agents targeting the intestinal barrier function have not been fully developed previously, in part due to a delay in understanding the mechanism underlying the impairment of the intestinal barrier function. In the present study, we hypothesized that CLDN2, a characteristic component of leaky TJ seals, might be an effective target through which to increase TJ integrity for IBD therapy. We consequently generated an $\mathrm{mAb}$ that recognized the first extracellular loop domain of CLDN2; the $\mathrm{mAb}$ restored the TJ seal function in cultures of TNF- $\alpha$-treated cells.

Infliximab and adalimumab, commercially available anti-TNF- $\alpha$ mAbs, prevent the intestinal barrier dysfunction due to TNF- $\alpha$ treatment, which increases the expression of CLDN2 (Fischer et al., 2013). Butyrate, a short-chain fatty acid, prevents chemical-induced colitis, accompanied by downregulation of CLDN2 (Vieira et al., 2012). Berberine, a plant alkaloid, is a traditional Eastern medicine for the treatment of diarrhea and gastroenteritis (Rosenthal et al., 2010); like butyrate, berberine downregulates CLDN2, the effect that is associated with the antidiarrheal properties of berberine. These findings strongly support our data regarding the use of an anti-CLDN2 mAb to treat the TNF- $\alpha$-induced dysfunction of TJ seals in the intestinal epithelium of patients with IBDs. However, butyrate downregulated 161 genes, including CLDN2 (Vieira et al., 2012), and berberine inhibited the PI3KAkt signaling pathway, which regulates CLDN2 expression (Rosenthal et al., 2010). Therefore, butyrate and berberine are not specific inhibitors of CLDN2, and, as such, their use for IBD therapy might cause unexpected adverse effects. In addition, CLDN2 was detectable in the colons of IBD patients but not in the colons of healthy people (Heller et al., 2005; Zeissig et al., 2007; Oshima et al., 2008). Accordingly, one key therapeutic benefit of an anti-CLDN2 mAb is its apparent specificity.

CLDN2 forms paracellular channels for cations and water (Amasheh et al., 2002, 2010). Incorporation of CLDN2 into TJ strands does not change their number but rather makes them discontinuous, resulting in the formation of TJ seals that are leaky to solutes (Furuse et al., 2001). CLDN2 forms homodimers with adjacent CLDN2 or heterodimers with CLDN3, but CLDN2 does not interact with CLDN1 (Furuse et al., 1999). The trans-interaction in CLDN2 homodimers is mediated by the first extracellular domains of each unit through electrostatic interactions with D65 and weaker interactions with negatively charged $\pi$ electrons of the Y67 aromatic residue (Lim et al., 2008). Our anti-CLDN2 mAb $1 \mathrm{~A} 2$ recognized the first extracellular domain of CLDN2. Given these findings, the binding of an anti-CLDN2 mAb to CLDN2 might prevent cis- and trans-

(after treatment with anti-CLDN2 mAb, black bars). Data are shown as the mean \pm S.D. $(n=3) . * P<0.05$ (Tukey's test). (C) Additive effects of anti-TNF- $\alpha$ mAb and anti-CLDN2 mAb on TJ integrity disrupted by TNF- $\alpha$ treatment. When TEER values had reached a plateau (approximately 10 days after cells were seeded on the Transwell membrane), the cells were treated with vehicle (medium) or TNF- $\alpha$ (10 ng/ml) for 24 hours. Then the cells were treated with anti-CLDN2 mAb 1A2 or rat IgG $(10 \mu \mathrm{g} / \mathrm{ml})$ and vehicle (PBS) or anti-TNF- $\alpha$ mAb [infliximab (Inf) or adalimumab (Ada), $10 \mu \mathrm{g} / \mathrm{ml}$ ] for 48 hours. TEER values were measured before and after TNF- $\alpha$ treatment ( -24 and 0 hours, respectively) and after anti-CLDN2 mAb treatment (48 hours). Data are shown as the mean \pm S.D. $(n=3)$. $* P<0.05$ (Tukey's test). 
interactions of CLDN2, thus attenuating the formation of leaky TJ seals.

CLDN2 is expressed in normal tissues, including liver, kidney, and small intestine (specifically the crypt-villus axis in humans), but not in the human colon (Furuse et al., 1998a; Enck et al., 2001; Kiuchi-Saishin et al., 2002; Lameris et al., 2013). CLDN2-deficient mice are normal in appearance, activity, growth, and behavior and lack functional and histologic abnormalities of liver, kidney, and small intestine (Muto et al., 2010; Tamura et al., 2011). Therefore, CLDN2 might be a safe target for IBD therapy.

In conclusion, we have generated an mAb that recognizes the first extracellular loop domain of CLDN2; this antiCLDN2 mAb ameliorated TNF- $\alpha$-induced loss of intestinal barrier function in vitro. CLDN2 binders might be a potent therapeutic option for IBD therapy to enhance the intestinal mucosal barrier.

\section{Acknowledgments}

We thank Dr. K. Endo and all of the members of our laboratory for their technical support, instruction, and useful comments.

\section{Authorship Contributions}

Participated in research design: Takigawa, Iida, Suzuki, Tada, Kunisawa, and Kondoh.

Conducted experiments: Takigawa, Iida, Nagase, and Suzuki. Contributed new reagents or analytic tools: Watari and Tada.

Performed data analysis: Takigawa, Iida, Suzuki, Tada, Okada,

Doi, Fukasawa, Yagi, Kunisawa, and Kondoh.

Contributed to the writing of the manuscript: Takigawa, Iida, Suzuki, Watari, Tada, Okada, Doi, Fukasawa, Yagi, Kunisawa, and Kondoh.

\section{References}

Amasheh M, Fromm A, Krug SM, Amasheh S, Andres S, Zeitz M, Fromm M, and Schulzke JD (2010) TNFalpha-induced and berberine-antagonized tight junction barrier impairment via tyrosine kinase, Akt and NFkappaB signaling. $J$ Cell Sci 123:4145-4155.

Amasheh M, Grotjohann I, Amasheh S, Fromm A, Söderholm JD, Zeitz M, Fromm M, and Schulzke JD (2009) Regulation of mucosal structure and barrier function in rat colon exposed to tumor necrosis factor alpha and interferon gamma in vitro: a novel model for studying the pathomechanisms of inflammatory bowel disease cytokines. Scand J Gastroenterol 44:1226-1235.

Amasheh S, Meiri N, Gitter AH, Schöneberg T, Mankertz J, Schulzke JD, and Fromm M (2002) Claudin-2 expression induces cation-selective channels in tight junctions of epithelial cells. J Cell Sci 115:4969-4976.

Bravatà I, Allocca M, Fiorino G, and Danese S (2015) Integrins and adhesion molecules as targets to treat inflammatory bowel disease. Curr Opin Pharmacol 25 $67-71$

Bürgel N, Bojarski C, Mankertz J, Zeitz M, Fromm M, and Schulzke JD (2002) Mechanisms of diarrhea in collagenous colitis. Gastroenterology 123:433-443.

de Souza HS and Fiocchi C (2016) Immunopathogenesis of IBD: current state of the art. Nat Rev Gastroenterol Hepatol 13:13-27.

Enck AH, Berger UV, and Yu AS (2001) Claudin-2 is selectively expressed in proximal nephron in mouse kidney. Am J Physiol Renal Physiol 281:F966-F974.

Fischer A, Gluth M, Pape UF, Wiedenmann B, Theuring F, and Baumgart DC (2013) Adalimumab prevents barrier dysfunction and antagonizes distinct effects of TNF- $\alpha$ on tight junction proteins and signaling pathways in intestinal epithelial cells. Am J Physiol Gastrointest Liver Physiol 304:G970-G979.

Fukasawa M, Nagase S, Shirasago Y, Iida M, Yamashita M, Endo K, Yagi K, Suzuki T, Wakita T, Hanada K, et al. (2015) Monoclonal antibodies against extracellular domains of claudin-1 block hepatitis $\mathrm{C}$ virus infection in a mouse model. $J$ Virol 89: $4866-4879$

Furuse M, Fujita K, Hiiragi T, Fujimoto K, and Tsukita S (1998a) Claudin-1 and -2 novel integral membrane proteins localizing at tight junctions with no sequence similarity to occludin. $J$ Cell Biol 141:1539-1550.

Furuse M, Furuse K, Sasaki H, and Tsukita S (2001) Conversion of zonulae occludentes from tight to leaky strand type by introducing claudin-2 into Madin-Darby canine kidney I cells. J Cell Biol 153:263-272.

Furuse M, Sasaki H, Fujimoto K, and Tsukita S (1998b) A single gene product, claudin-1 or -2 , reconstitutes tight junction strands and recruits occludin in fibroblasts. J Cell Biol 143:391-401.

Furuse M, Sasaki H, and Tsukita S (1999) Manner of interaction of heterogeneous claudin species within and between tight junction strands. J Cell Biol 147:891-903.

Graham WV, Wang F, Clayburgh DR, Cheng JX, Yoon B, Wang Y, Lin A, and Turner JR (2006) Tumor necrosis factor-induced long myosin light chain kinase transcription is regulated by differentiation-dependent signaling events.
Characterization of the human long myosin light chain kinase promoter. $J$ Biol Chem 281:26205-26215.

Heller F, Florian P, Bojarski C, Richter J, Christ M, Hillenbrand B, Mankertz J, Gitter AH, Bürgel N, Fromm M, et al. (2005) Interleukin-13 is the key effector Th2 cytokine in ulcerative colitis that affects epithelial tight junctions, apoptosis, and cell restitution. Gastroenterology 129:550-564.

Heller F, Fromm A, Gitter AH, Mankertz J, and Schulzke JD (2008) Epithelial apoptosis is a prominent feature of the epithelial barrier disturbance in intestina inflammation: effect of pro-inflammatory interleukin-13 on epithelial cell function. Mucosal Immunol 1 (Suppl. 1):S58-S61.

Hering NA, Fromm M, and Schulzke JD (2012) Determinants of colonic barrier function in inflammatory bowel disease and potential therapeutics. $J$ Physiol 590: 1035-1044.

Kiuchi-Saishin Y, Gotoh S, Furuse M, Takasuga A, Tano Y, and Tsukita S (2002) Differential expression patterns of claudins, tight junction membrane proteins, in mouse nephron segments. $J$ Am Soc Nephrol 13:875-886.

Kuwada M, Chihara Y, Luo Y, Li X, Nishiguchi Y, Fujiwara R, Sasaki T, Fujii K, Ohmori H, Fujimoto K, et al. (2015) Pro-chemotherapeutic effects of antibody against extracellular domain of claudin-4 in bladder cancer. Cancer Lett 369: $212-221$

Lameris AL, Huybers S, Kaukinen K, Mäkelä TH, Bindels RJ, Hoenderop JG, and Nevalainen PI (2013) Expression profiling of claudins in the human gastrointestinal tract in health and during inflammatory bowel disease. Scand $J$ Gastroenterol 48:58-69.

Li X, Iida M, Tada M, Watari A, Kawahigashi Y, Kimura Y, Yamashita T, IshiiWatabe A, Uno T, Fukasawa M, et al. (2014) Development of an anti-claudin-3 and -4 bispecific monoclonal antibody for cancer diagnosis and therapy. J Pharmacol Exp Ther 351:206-213.

Lim TS, Vedula SR, Hunziker W, and Lim CT (2008) Kinetics of adhesion mediated by extracellular loops of claudin-2 as revealed by single-molecule force spectroscopy. J Mol Biol 381:681-691.

Luettig J, Rosenthal R, Barmeyer C, and Schulzke JD (2015) Claudin-2 as a mediator of leaky gut barrier during intestinal inflammation. Tissue Barriers 3:e977176.

Ma TY, Boivin MA, Ye D, Pedram A, and Said HM (2005) Mechanism of TNF-alpha modulation of Caco-2 intestinal epithelial tight junction barrier: role of myosin light-chain kinase protein expression. Am J Physiol Gastrointest Liver Physiol 288 : G422-G430.

Mankertz J, Amasheh M, Krug SM, Fromm A, Amasheh S, Hillenbrand B, Tavalali S, Fromm M, and Schulzke JD (2009) TNFalpha up-regulates claudin-2 expression in epithelial HT-29/B6 cells via phosphatidylinositol-3-kinase signaling. Cell Tissue Res 336:67-77.

Meunier V, Bourrié M, Berger Y, and Fabre G (1995) The human intestinal epithelial cell line Caco-2; pharmacological and pharmacokinetic applications. Cell Biol Toxicol 11:187-194.

Mosley M, Knight J, Neesse A, Michl P, Iezzi M, Kersemans V, and Cornelissen B (2015) Claudin-4 SPECT imaging allows detection of aplastic lesions in a mouse model of breast cancer. $J$ Nucl Med 56:745-751.

Muto S, Hata M, Taniguchi J, Tsuruoka S, Moriwaki K, Saitou M, Furuse K, Sasaki H, Fujimura A, Imai M, et al. (2010) Claudin-2-deficient mice are defective in the leaky and cation-selective paracellular permeability properties of renal proximal tubules. Proc Natl Acad Sci USA 107:8011-8016.

Nakajima M, Nagase S, Iida M, Takeda S, Yamashita M, Watari A, Shirasago Y, Fukasawa M, Takeda H, Sawasaki T, et al. (2015) Claudin-1 binder enhances epidermal permeability in a human keratinocyte model. J Pharmacol Exp Ther 354:440-447.

Neesse A, Hahnenkamp A, Griesmann H, Buchholz M, Hahn SA, Maghnouj A Fendrich V, Ring J, Sipos B, Tuveson DA, et al. (2013) Claudin-4-targeted optical imaging detects pancreatic cancer and its precursor lesions. Gut 62:1034-1043.

Noth R, Stüber E, Häsler R, Nikolaus S, Kühbacher T, Hampe J, Bewig B, Schreiber $\mathrm{S}$, and Arlt A (2012) Anti-TNF- $\alpha$ antibodies improve intestinal barrier function in Crohn's disease. J Crohn's Colitis 6:464-469.

Oshima T, Miwa H, and Joh T (2008) Changes in the expression of claudins in active ulcerative colitis. J Gastroenterol Hepatol 23 (Suppl 2):S146-S150.

Podolsky DK (2002) Inflammatory bowel disease. N Engl J Med 347:417-429.

Powell DW (1981) Barrier function of epithelia. Am J Physiol 241:G275-G288.

Prasad S, Mingrino R, Kaukinen K, Hayes KL, Powell RM, MacDonald TT, and Collins JE (2005) Inflammatory processes have differential effects on claudins 2, 3 and 4 in colonic epithelial cells. Lab Invest 85:1139-1162.

Rosenthal R, Milatz S, Krug SM, Oelrich B, Schulzke JD, Amasheh S, Günzel D, and Fromm M (2010) Claudin-2, a component of the tight junction, forms a paracellular water channel. J Cell Sci 123:1913-1921.

Schmitz H, Fromm M, Bentzel CJ, Scholz P, Detjen K, Mankertz J, Bode H, Epple HJ, Riecken EO, and Schulzke JD (1999) Tumor necrosis factor-alpha (TNFalpha) regulates the epithelial barrier in the human intestinal cell line HT-29/B6. J Cell Sci 112:137-146.

Staehelin LA (1974) Structure and function of intercellular junctions. Int Rev Cytol 39:191-283.

Suenaert P, Bulteel V, Lemmens L, Noman M, Geypens B, Van Assche G, Geboes K, Ceuppens JL, and Rutgeerts P (2002) Anti-tumor necrosis factor treatment restores the gut barrier in Crohn's disease. Am J Gastroenterol 97:2000-2004.

Suzuki T, Yoshinaga N, and Tanabe S (2011) Interleukin-6 (IL-6) regulates claudin-2 expression and tight junction permeability in intestinal epithelium. $J$ Biol Chem 286:31263-31271.

Takahashi A, Saito Y, Kondoh M, Matsushita K, Krug SM, Suzuki H, Tsujino H, Li X, Aoyama H, Matsuhisa K, et al. (2012) Creation and biochemical analysis of a broad-specific claudin binder. Biomaterials 33:3464-3474.

Tamura A, Hayashi H, Imasato M, Yamazaki Y, Hagiwara A, Wada M, Noda T, Watanabe M, Suzuki Y, and Tsukita S (2011) Loss of claudin-15, but not claudin-2, causes $\mathrm{Na}+$ deficiency and glucose malabsorption in mouse small intestine. Gastroenterology 140:913-923. 
Van Itallie CM and Anderson JM (2014) Architecture of tight junctions and principles of molecular composition. Semin Cell Dev Biol 36:157-165.

Vieira EL, Leonel AJ, Sad AP, Beltrão NR, Costa TF, Ferreira TM, Gomes-Santo AC, Faria AM, Peluzio MC, Cara DC, et al. (2012) Oral administration of sodium butyrate attenuates inflammation and mucosal lesion in experimental acute ulcerative colitis. J Nutr Biochem 23:430-436.

Wang F, Graham WV, Wang Y, Witkowski ED, Schwarz BT, and Turner JR (2005) Interferon-gamma and tumor necrosis factor-alpha synergize to induce intestinal epithelial barrier dysfunction by up-regulating myosin light chain kinase expression. Am J Pathol 166:409-419.
Zeissig S, Bürgel N, Günzel D, Richter J, Mankertz J, Wahnschaffe U, Kroesen AJ, Zeitz M, Fromm M, and Schulzke JD (2007) Changes in expression and distribution of claudin 2,5 and 8 lead to discontinuous tight junctions and barrier dysfunction in active Crohn's disease. Gut 56:61-72.

Address correspondence to: Dr. Masuo Kondoh, Graduate School of Pharmaceutical Sciences, Osaka University, Suita, Osaka 565-0871, Japan. E-mail: masuo@phs.osaka-u.ac.jp 